\title{
Electromyographic Study on Reflex Activity in Abdominal Wall Muscles and Diaphragm Following Pelvic Afferent Excitation in Cats
}

\author{
By \\ Shinjiro Yamamoto, Kimpei Araki and Makoto Kikuchi \\ From the Department of Surgery, School of Medicine, \\ University of Kanazawa, Kanazawa
}

(Received for publication, August 30, 1961)

\section{INTRODUCTION}

The so-called abdominal rigidity due to irritation of the abdominal viscera was noted by Fulton ${ }^{5}$ ) as a flexion reflex. As to the afferent sources to produce the abdominal rigidity or the reflex contraction of the abdominal muscles, the splanchnic and the somatic nerves innervating the abdominal wall have been studied, and their reflex centers are proved to be involved in the spinal cord ${ }^{21}$, 3),4), 1),8). However, Yamamoto, Araki and Kikuchi' ${ }^{9}$ demonstrated that electrical stimulation of the pelvic nerve induces a marked increase of the intraabdominal pressure which is caused by simultaneous contractions of the whole abdominal muscles and the diaphragm, and that the center of this reflex arc is located in the lower medulla. This reflex activity has been designated as "pelvicoabdominal reflex".

In this paper we describe electromyographic responses recorded from the muscles of trunk and abdomen as well as from the diaphragm in the pelvicoabdominal reflex.

\section{METHODS}

The experiments were carried out on 20 cats weighing $2.5-3.2 \mathrm{~kg}$, anesthetized with intravenous injection of thiamylal sodium. The trachea was cannulated. Through a median incision of the abdomen, the pelvic nerves were exposed on both sides and a pair of indwelling electrodes were attached on the nerves at a level of the lateral wall of the rectum. In one experiment, stimulation was applied to the splanchnic nerve which was exposed retroperitoneally on the left side. The stimulating electrodes of the splanchnic nerve were attached just proximal to the celiac ganglion. A balloon, connected with a mechano-electrical transducer, was inserted into the abdominal cavity and changes of the intra-abdominal 
pressure were recorded on films by an oscilloscope. The pelvic nerve was stimulated with square waves of $0.4 \mathrm{msec}$ duration through an isolation unit. Muscle activities were recorded from various muscles by the use of a concentric needle electrode connected to a CR-coupled amplifier and an oscilloscope. To record from the diaphragm, the electrode was inserted into its costal part through a lower intercostal space. To record from the muscles of trunk and abdomen, the skin overlying the aimed area was removed to expose the muscle to be studied.

\section{RESULTS}

Repetitive stimulation of the central end of the pelvic nerve provoked a tonic contraction of the abdominal wall resulting in an increase of the intra-abdominal pressure. By an inspection, the whole abdominal muscles as well as the diaphragm were found to be involved in the reflex activities. As reported by Yamamoto et $\left.a l .{ }^{9}\right)$ previously, a maximal tonic contraction of the abdominal wall was obtained by repetitive stimulation at approximately $50 \mathrm{cps}$ using the intensity sufficient to activate the A gamma-delta fibers of the pelvic nerve. Using such stimulation parameters, myographic responses were examined in some muscles participating in the reflex. Sample records are shown in Fig. 1.
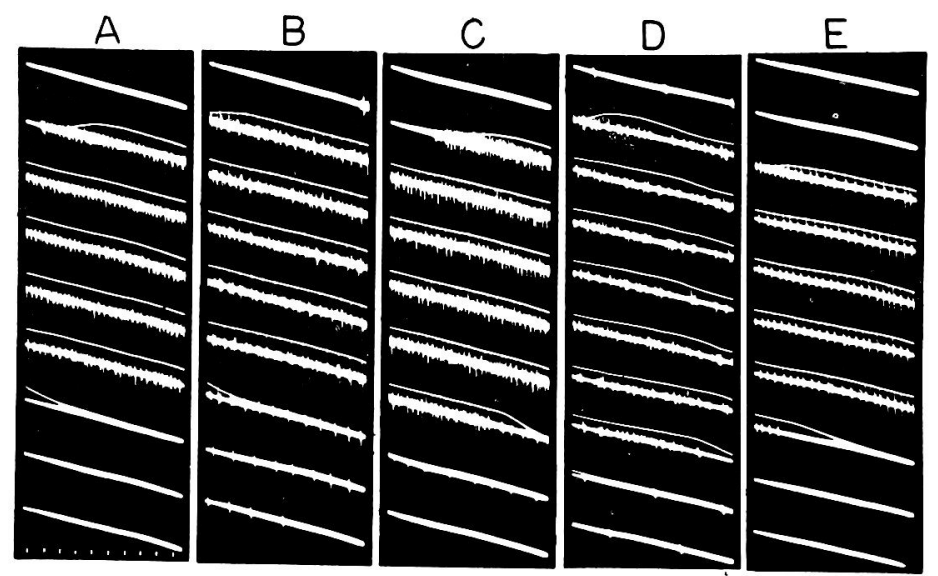

Fig. 1. Electromyographic records from muscles participating in pelvicoabdominal reflex. Right pelvic nerve was stimulated with square waves of 0.4 msec pulse duration at 50 cps for about 5 sec.
A, left rectus abdominis.
B, right rectus abdominis.
C, right obliquus externus. D, pars costalis of diaphragm. E, 9th intercostal muscle. Strength of intra-abdominal pressure change is indicated by distance between two beams. Recurrent sweep. Time, 0.1 sec.

It is found that excitation of the pelvic afferents provoked the discharges not only in the muscles surrounding the abdominal cavity such as the abdominal wall muscles and the diaphragm, but also in the lower intercostal muscles. From the 
pectoralis major and latissimus dorsi, no response was obtained. In each muscle, there was encountered a slight difference in the response pattern according to the muscle unit examined. However, there was no evidence which permitted us to distinguish any particular muscles in the response type. As expected from inspection of the muscle contraction with naked eye, no appreciable laterality to the side of peripheral stimulation could be found in the distribution of the reflex activities even with the electromyographic method. The unit discharge of muscle did not show a strict correspondence to each stimulation pulse. The frequency and regularity of the discharge were also quite different between the units. Another notable feature of this reflex activity was a long lasting after-discharge. As exemplified by Fig. 1B, some units continued to discharge as long as $3 \mathrm{sec}$ after cessation of the stimulation.

\section{Effects of anesthetic drug on reflex activity.}

General anesthesia impairs the reflex. In a deeply anesthetized state at which spontaneous respiration was on the verge of stopping, the reflex abdominal contraction could not be produced. The group of abdominal wall muscles was found more susceptible to anesthesia than the diaphragm. As to the abdominal muscles, spontaneous discharges or periodic discharges probably relating with

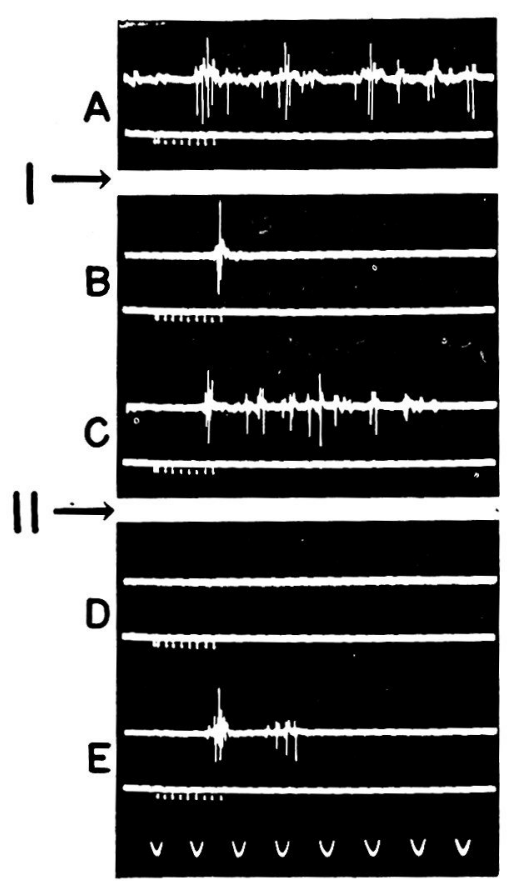

$50 \mathrm{~ms}$
Fig. 2. Effect of intravenous application of thiamylal sodium on reflex discharge of external oblique muscle. A, control record. B, immediately after intravenous injection of $2 \mathrm{mg} / \mathrm{kg}$ of drug (I). C, $20 \mathrm{sec}$ after B. D, after additional application of $2 \mathrm{mg} / \mathrm{kg}$ of drug (II). $\mathrm{E}$, 60 sec after $D$.

Upper trace, electromyographic record. Bottom trace, stimulus marks. Square waves of $0.4 \mathrm{msec}$ pulse duration and 100 cps frequency were applied for 0.08 sec. Time, 50 msec. 
the respiratory movement were more susceptible to anesthesia than the reflexly evoked discharges.

The example shown in Fig. 2 demonstrates an effect of thiamylal sodium on the reflex activity of the external oblique muscle. The recording was started at a light anesthesia just stopping the spontaneous discharges of the muscle. The unit, though failing to respond to single shock stimulation, could be activated by a train of stimulation impulses. Therefore, $100 \mathrm{cps}$ stimulation was applied to the pelvic nerve during $0.08 \mathrm{sec}$ in each record. The control record (Fig. 2-A) showed that the earliest group of the discharges was followed by the delayed discharges lasting approximately $300 \mathrm{msec}$. An intravenous injection of $2 \mathrm{mg} / \mathrm{kg}$ of the drug eliminated the delayed discharges and reduced the number of spikes in the earliest group (B). It was also noted that the response time, which was measured $60 \mathrm{msec}$ in the control record, was prolonged up to $80 \mathrm{msec}$ by the application of the drug. The delayed discharges of the response came back $20 \mathrm{sec}$ after the injection (C). An additional injection of $2 \mathrm{mg} / \mathrm{kg}$ of the drug at this stage eliminated the whole response completely (D). The early discharges came back $20 \mathrm{sec}$ thereafter, and it took $60 \mathrm{sec}$ for the delayed discharges to reappear $(\mathrm{E})$.

\section{Summating action of repetitive stimuli.}

Muscle units participating in the abdominal reflex contraction showed some individual properties in the response pattern. When a maximal stimulation intensity was used, some units could be activated by a single shock stimulus, while others not. The units of the latter type could be activated only when stimulation impulses were applied repetitively at a certain frequency.

In the experiment shown in Fig. 3, the unit became activated by double shocks with shock interval of $3-30$ msec. The stimulation effect of the double shocks was maximal when the shock interval was 10-20 msec. The double shocks with too short or too long interval failed to activate this unit.

In the case shown in Fig. 4, an effect of lengthening the shock interval of the double shocks was studied with a unit which could be activated by single shock stimulation. Stimulation and recording were made every four sec. An augmentation of the reflex activity appeared with the shock interval longer than 1 msec, a maximal response being obtained with the interval of 10-20 msec. When the interval exceeded $40 \mathrm{msec}$, a separation of the response could be seen. However, it was noted that the activity elicited by the second shock was much bigger than that by the first shock. This may mean that the first shock produces a facilitatory state in the reflex center. Such a facilitatory state appeared to last approximately $0.5 \mathrm{sec}$. Another notable finding in this experiment is that the muscle unit responded to a single shock with only one spike at the earlier stage of the series of recording (Fig. 4A-B), but when the experiment continued for a 


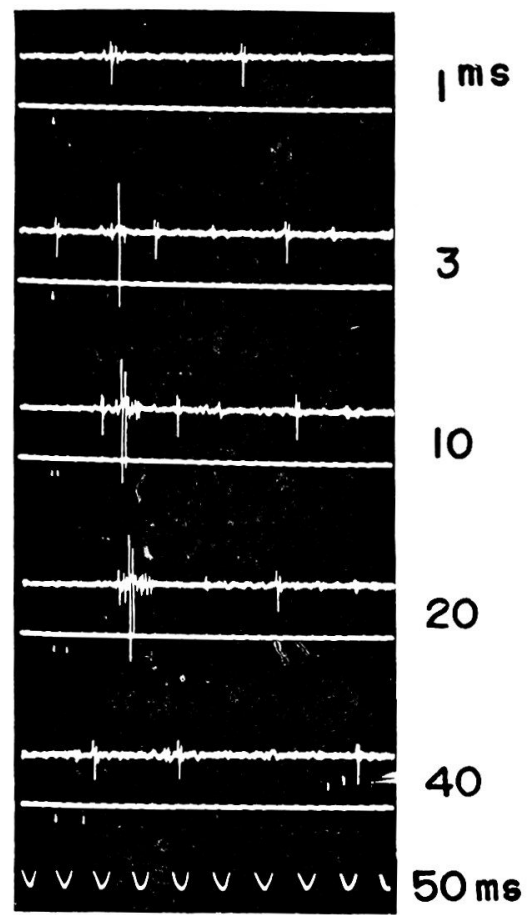

Fig. 3.

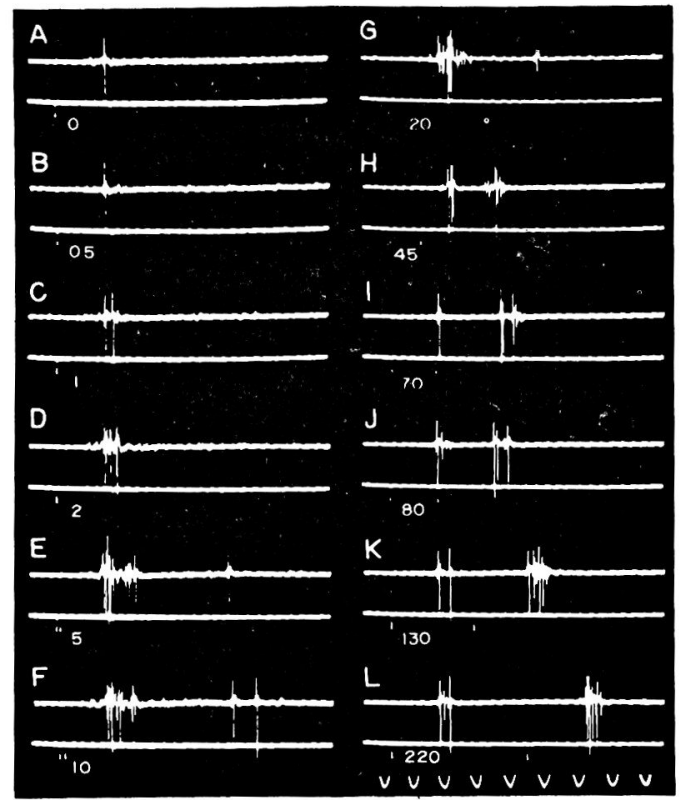

Fig. 4.

Fig. 3. Electromyographic record from diaphragm. Double pulses with various intervals were applied to pelvic nerve. Numbers in right column indicate pulse interval in msec. Time, 50 msec.

Fig. 4. Electromyographic record from external oblique muscle. Ipsilateral pelvic nerve was stimulated with double pulses every four seconds. Numbers below each record indicate pulse intervals in msec. In record $\mathrm{A}$, single pulse stimulation was used. Time, 50 msec.

considerable time, the same unit responded to the first pulse of each double shocks with multiple spikes (Fig. 4H-L). This may be considered to be due to increase of the central responsiveness which was brought about by repeated application of the double shocks to the pelvic nerve. Thus it is suggested that the medullary center of the pelvico-abdominal reflex has a strong tendency to enhance its excitability by successive inflows of the reflexogenous sensory impulses.

In the experiment shown in Fig. 5, trains of stimulus impulses with various frequencies were applied for $0.2 \mathrm{sec}$ to the pelvic nerve every five sec, and the reflex activities were recorded from the ipsilateral external oblique muscle. In the resting state there were found spontaneous discharges of small spike in a tonic manner. The discharge pattern to $10 \mathrm{cps}$ stimulation was nearly the same as that in the resting state. When the stimulation frequency exceeded $20 \mathrm{cps}$, the reflexly evoked big spikes appeared with the contraction of the muscle. At 


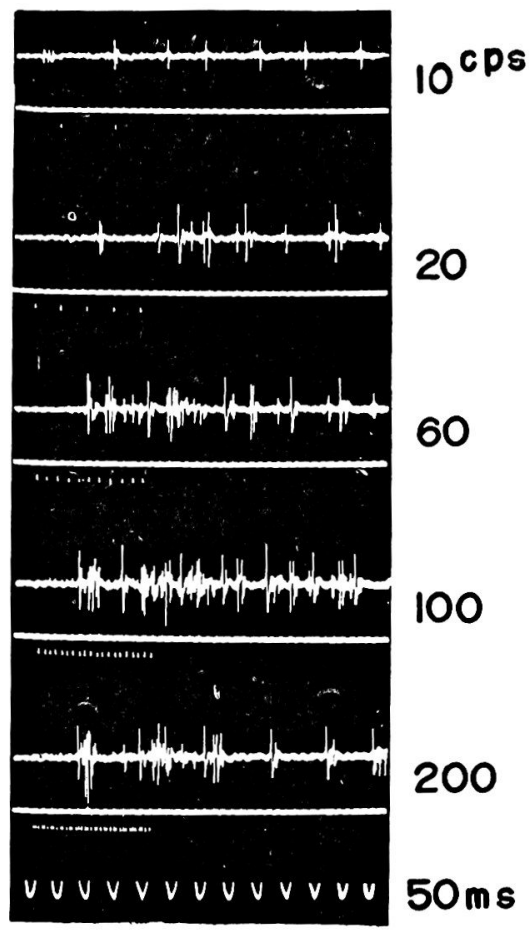

Fig. 5.

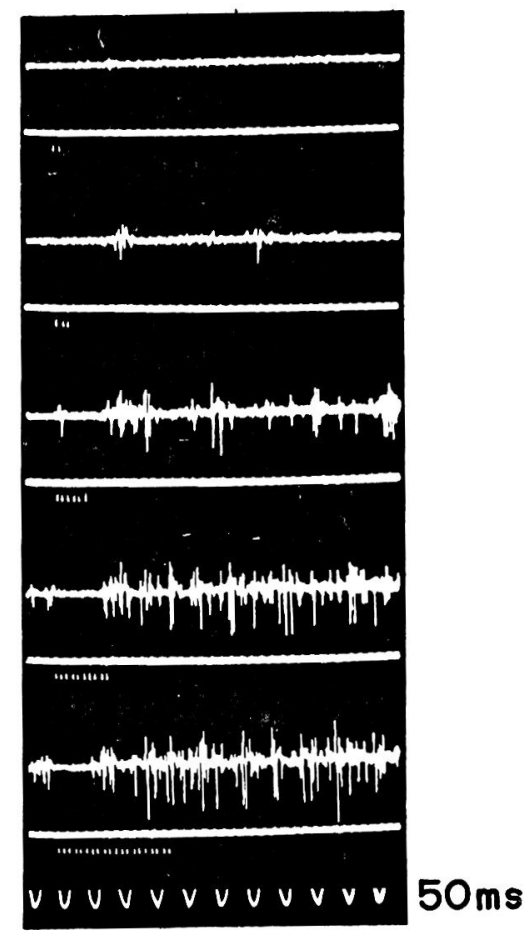

Fig. 6.

Fig. 5. Electromyographic record from external oblique muscle. Ipsilateral pelvic nerve was stimulated for $0.2 \mathrm{sec}$ with pulses at varying frequencies. Numbers in right column indicate frequency of stimulation pulses. Time, $50 \mathrm{msec}$.

Fig. 6. Electromyographic record from rectus abdominis. Ipsilateral pelvic nerve was stimulated with pulses of $100 \mathrm{cps}$ for varying durations. Time, $50 \mathrm{msec}$.

that time, the tonic spontaneous discharges disappeared in the interval period between each reflex contraction of the muscle. Increasing the stimulation frequency the response time was reduced. The muscle activity also augmented with increased stimulation frequency, but its maximum was obtained at about the frequency of $100 \mathrm{cps}$. When the stimulation frequency increased up to $200 \mathrm{cps}$, the early discharges became more marked with the following delayed discharges more or less suppressed.

Fig. 6 shows an example in which the effects of varying the duration of stimulation were examined keeping the frequency of repetitive impulses at $100 \mathrm{cps}$. The unit could be activated by the stimulation with more than 3 pulses. The muscle discharges became more marked as the duration of stimulation was prolonged, probably owing to the recruitment mechanism in the reflex center. It was frequently observed that the late recruited discharge was bigger in the spike height. It remains obscure if the bigger spike is produced by 
synchronization of many small discharges or it is an independent unit which was silent otherwise.

\section{Latency of reflexes.}

A considerable variation was observed in the latency of the pelvico-abdominal reflex even with the same muscle unit. Its cause appeared to be the depth of anesthesia, the strength of stimulation and the facilitatory background of the reflex arcs. The experiment in Fig. 2 showed that a shot of anesthetic drug prolonged the latency as much as by $20 \mathrm{msec}$. When the central excitatory state was supposed to be high as evidenced by the existence of the spontaneous muscle discharges related to the spontaneous respiration, the response time was definitely smaller.

The muscle units which could be activated by single shock stimulation to the pelvic nerve appeared to be smaller in number than those activated by repetitive stimulation. When repetitive stimulation was used, it was found that the higher the frequency, the shorter the response time, but no appreciable reduction in the response time could be found with stimulation frequencies higher than 100 cps. The latencies of units were measured by the time interval between the first pulse of stimulation volley of $100 \mathrm{cps}$ and the first spike of response discharge. The results were $60-120 \mathrm{msec}$ for 34 units in the external oblique, 80-100 msec for 7 units in the rectus abdominis and 60-80 msec for 7 units in the diaphragm.

As a supplement to the above data, we measured the response time in 7 units of the external oblique muscle following stimulation of the ipsilateral splanchnic nerve. Each unit responded to single shock stimulation and the latencies distributed in the range of $10-17 \mathrm{msec}$.

\section{DISCUSSION}

The pelvico-abdominal reflex is complete only when the lower medulla is intact, being a multisynaptic reflex projecting into the abdominal muscles, the lower intercostal muscles and the diaphragm. This makes a marked contrast to the reflex activities in the above muscles following stimulation of the splanchnic nerve or the abdominal somatic nerve branches. Downman ${ }^{3}$ showed that latencies of responses recorded from the $\mathrm{Th}_{11}$ ventral root following splanchnic nerve stimulation were $5.7-7.2 \mathrm{msec}$. This value appears, when the time is allowed for peripheral conduction, to be comparable to the response time of 10-17 msec in our results which were obtained from the external oblique muscle following splanchnic nerve stimulation. Chennels and Floyd 's showed that the latencies of reflex response of stimulation of the cutaneous branch of the nerves $\mathrm{Th}_{13}-\mathrm{L}_{3}$ in cats were 10-15 msec for the abdominal muscles. Therefore, the values obtained by the splanchnic nerve stimulation are quite comparable to those by 
the abdominal somatic nerve stimulation. On the contrary, the response time of abdominal muscles to pelvic nerve stimulation was found to exceed $60 \mathrm{msec}$. This was true not only for the abdominal wall muscles but also for the diaphragm. Such long latencies appear to be dependent not only upon the long reflex arcs, but also upon the long central delay. Calma" showed that a single afferent volley of somatic origin causes the efferent discharges in the phrenic nerve with the nuclear delay of 7-10 msec in the respiratory center of the medulla. Since the pelvico-abdominal reflex has its center in the medulla near the respiratory center, ${ }^{9)}$ its nuclear delay is supposedly as large as in the respiratory center.

A notable finding in the abdominal contraction responding to the pelvic nerve stimulation was a long lasting facilitatory effect of stimulation. This effect appears to present a basis for the phenomenon of recruitment of abdominal contraction which is produced by continuous stimulation of the pelvic nerve with low frequencies such as $10-50 \mathrm{cps}^{\circ}{ }^{2}$ With repetitive stimulation at frequencies greater than $100 \mathrm{cps}$, there was a reduction in the reflex muscle discharges, depressing particularly the delayed discharge. This phenomenon is considered to be the Wedensky inhibition.

In a study of the action of the anesthetic drug on muscle discharges, it was found that spontaneous firing of the muscle was found to be most sensitive to anesthesia. Of the train of reflex discharges of the abdominal muscle, the delayed discharge is more susceptible than the earlier phase of the discharges, though some prolongation of the response time is observed. If the delayed discharge is presumed to be activity relayed through a complicated neuronal networks, their vulnerability to anesthesia may be interpreted to mean that the neurons with more synaptic relays are more sensitive to anesthesia. Concerning the cells of the somatosensory cortex and the brain stem in cats, Yamamoto and Schaeppi ${ }^{10)}$ reached the same conclusion.

In a certain depth of anesthesia, the pelvico-abdominal reflex could be eliminated while the abdominal contraction following splanchnic nerve stimulation persisted. This may show that the pelvico-abdominal reflex has a more complicated neuronal organization than the splanchnico-abdominal reflex. It is generally known that the respiratory activity of the intercostal muscles is more susceptible to deep anesthesia than that of the diaphragm. ${ }^{6}$ ) In the pelvico-abdominal reflex it has been shown that the abdominal muscles are a more susceptible component to anesthesia than the diaphragm. This suggests that the synaptic organization for innervating the abdominal and intercostal muscles may be more complicated than for the diaphragm.

\section{SUMMARY}

The intra-abdominal pressure response following electrical stimulation of the central end of the pelvic nerve (pelvico-abdominal reflex) was studied by 
means of the electromyographic technique in anesthetized cats. The muscles of the abdominal wall and lower intercostal space, and the diaphragm were involved in the reflex activity. The response time distributed in the range of 60-120 msec.

The muscle units which could be activated by a single shock to the pelvic nerve were small in number relative to those which could be activated by repetitive stimulation. The summating effect of the double shock stimulus was seen at the shock intervals of 2-30 msec with a maximum at 10-20 msec intervals. A single shock to the pelvic nerve produced a facilitatory state in the reflex arcs lasting approximately $0.5 \mathrm{sec}$. Repeated stimulation with low frequency pulses appeared to build up an excitatory state which initiated a recruitment of muscle activity and a long lasting after-discharge.

Spontaneous firing of muscle activity was most sensitive to anesthesia. It was the first to be affected with a small dose of the drug while the reflex response to pelvic nerve stimulation still persisted. When the depth of anesthesia was increased to a certain extent, the train of reflex discharges was deprived of its delayed component with some prolongation of the response time. Among the group of muscles participating in the abdominal contraction, the abdominal wall muscles were more vulnerable component to anesthesia than the diaphragm.

\section{ACKNOWLEDGMENT}

We wish to express our appreciation to Dr. M. Urabe for his interest and encouragement. Our grateful thanks are also due to Dr. K. Iwama for his invaluable suggestions in regard to the manuscript.

\section{References}

1) Calma, I., J. Physiol., 1952, 117, 9.

2) Chennels, M. \& Floyd, W. F., J. Physiol. 1952, 118, 196.

3) Downman, C.B.B., J. Neurophysiol., 1955, 18, 217.

4) Downman, C.B.B. \& McSwiney, B.A., J. Physiol., 1946, 105, 80.

5) Fulton, J.F., Physiology of the Nervous System. New York, Oxford University Press, 1949.

6) Miller, A.H., J.A.M.A., 1925, 84, 201.

7) Miller, F.R., Am. J. Physiol., 1924, 71, 84.

8) Miller, F.R. \& Waud, R.A., Am. J. Physiol., 1925, 73, 329.

9) Yamamoto, S., Araki, K. \& Kikuchi, M., Exp. Neurol. 1961, 4, 345.

10) Yamamoto, S. \& Schaeppi, U., Electroenceph. clin. Neurophysiol., 1961, 13, 248. 